\title{
The impact of COVID-19 on short-term prognosis of ST-segment elevation myocardial infarction patients receiving primary percutaneous coronary intervention in Wuhan China
}

\author{
Qing Lu ${ }^{1, \dagger}$, Jiu-Long Wang ${ }^{2, \dagger}$, Zhi-Nan Chen $^{1}$, Wen-Bo Fu ${ }^{1}$, Hui-Jian Liu ${ }^{1}$, Shi-Fang Ding ${ }^{1, *}$ \\ ${ }^{1}$ Department of Cardiology, General Hospital of Central Theater Command, Wuhan, 430070 Hubei, P. R. China \\ ${ }^{2}$ The First School of Clinical Medicine, Southern Medical University, Guangzhou, 510515 Guangdong, P. R. China \\ *Correspondence: shifangding116@163.com (Shi-Fang Ding) \\ ${ }^{\dagger}$ These authors contributed equally.
}

DOI:10.31083/j.rcm.2021.01.243

This is an open access article under the CC BY 4.0 license (https://creativecommons.org/licenses/by/4.0/).

Submitted: 13 November 2020 Revised: 22 January 2021 Accepted: 24 January 2021 Published: 30 March 2021

ST-segment elevation myocardial infarction (STEMI) is a common cardiovascular emergency for which timely reperfusion therapies are needed to minimize myocardial necrosis. The aim of this study was to investigate the impact of the COVID-19 pandemic and reorganization of chest pain centers $(\mathrm{CPC})$ on the practice of primary percutaneous coronary intervention (PPCl) and prognosis of STEMI patients. This single-center retrospective survey included all patients with STEMI admitted to our CPC from January 22, 2020 to April 30, 2020 (during COVID-19 pandemic in Wuhan), compared with those admitted during the analogous period in 2019, in respect of important time points of $\mathrm{PPCl}$ and clinical outcomes of STEMI patients. In the present article, we observed a descending trend in STEMI hospitalization and a longer time from symptom onset to first medical contact during the COVID-19 pandemic as compared to the control period ( $4.35 \mathrm{~h}$ versus $2.58 \mathrm{~h}$ ). With a median delay of 17 minutes in the door to balloon time (D2B), the proportion of in-hospital cardiogenic shock was significantly higher in the COVID-19 era group ( $47.6 \%$ versus $19.5 \%$ ), and major adverse cardiac events (MACE) tend to increase in the 6-month follow-up period ( $14.3 \%$ versus $2.4 \%$ ). Although the reorganization of CPC may prolong the $\mathrm{D} 2 \mathrm{~B}$ time, immediate revascularization of the infarct-related artery could be offered to most patients within 90 minutes upon arrival. PPCI remained the preferred treatment for patients with STEMI during COVID-19 pandemic in the context of timely implementation and appropriate protective measures.

Keywords

COVID-19; STEMI; PPCI; Chest pain center; Prognosis

\section{Introduction}

Since the late December 2019, COVID-19 caused by a novel severe acute respiratory syndrome coronavirus (SARSCOV-2) has rapidly swept the world in a few months $[1,2]$. According to data from the World Health Organization, as of September 28, 2020, more than 32 million cases of coronavirus disease (COVID-19) have been confirmed globally, of which a cumulative total of nearly 1 million were fatal [3]. Numerous studies have reported on the transmission routes, clinicopathological characteristics, and prognostic factors of COVID-19 [4-8]. The COVID-19 outbreak has caused un- precedent healthcare and socioeconomic crisis worldwide, and greatly impacted the clinical practice of other diseases. Multiple investigators have reported a reduction of patients admitted for acute coronary syndrome (ACS) during the early days of COVID-19 pandemic [9]. A nationwide survey of China Chest Pain Center [10] showed that approximately $26 \%$ decrease in weekly number of STEMI admissions during COVID-19 outbreak (62\% drop in Hubei), and the proportion of patients with STEMI receiving PPCI dropped by $50 \%$ after the recommendation of modified treatment protocol (thrombolysis as the preferred reperfusion strategy for patients without testing of COVID-19 in epidemic area). Through this work, Xiang et al. revealed delayed time to primary reperfusion and higher rates of in-hospital mortality and heart failure for patients with STEMI admitted during COVID-19 outbreak, which indicated the importance of timely and effective reperfusion therapy. Gramegna et al. [11] proposed PPCI remained a safe and feasible procedure for STEMI during COVID-19 outbreak with adequate personal protective equipment, as none of healthcare workers in their cohort participated in catheterization procedures acquired nosocomial SARS-COV-2 infection. However, few studies have focused on the reperfusion timeliness and shortterm outcomes of patients receiving PPCI during the pandemic.

In late January 2020, the CPC of our medical center (regional referral hub located in Wuhan for cardiovascular emergencies) adjusted the diagnostic and therapeutic processes for STEMI patients and the personal protective level of medical workers to ensure timely reperfusion and reduce cross-infection risk of SARS-COV-2. Under the level-3 protection, PPCI was conducted in an isolated cardiac catheterization laboratory (CCL) and all associated staff completed pre-training on the correct and appropriate use of personal protective equipment (PPE). In the present study, we retrospectively analyzed the data from our single-center to evaluate the impact of COVID-19 and reorganization of CPC ser- 


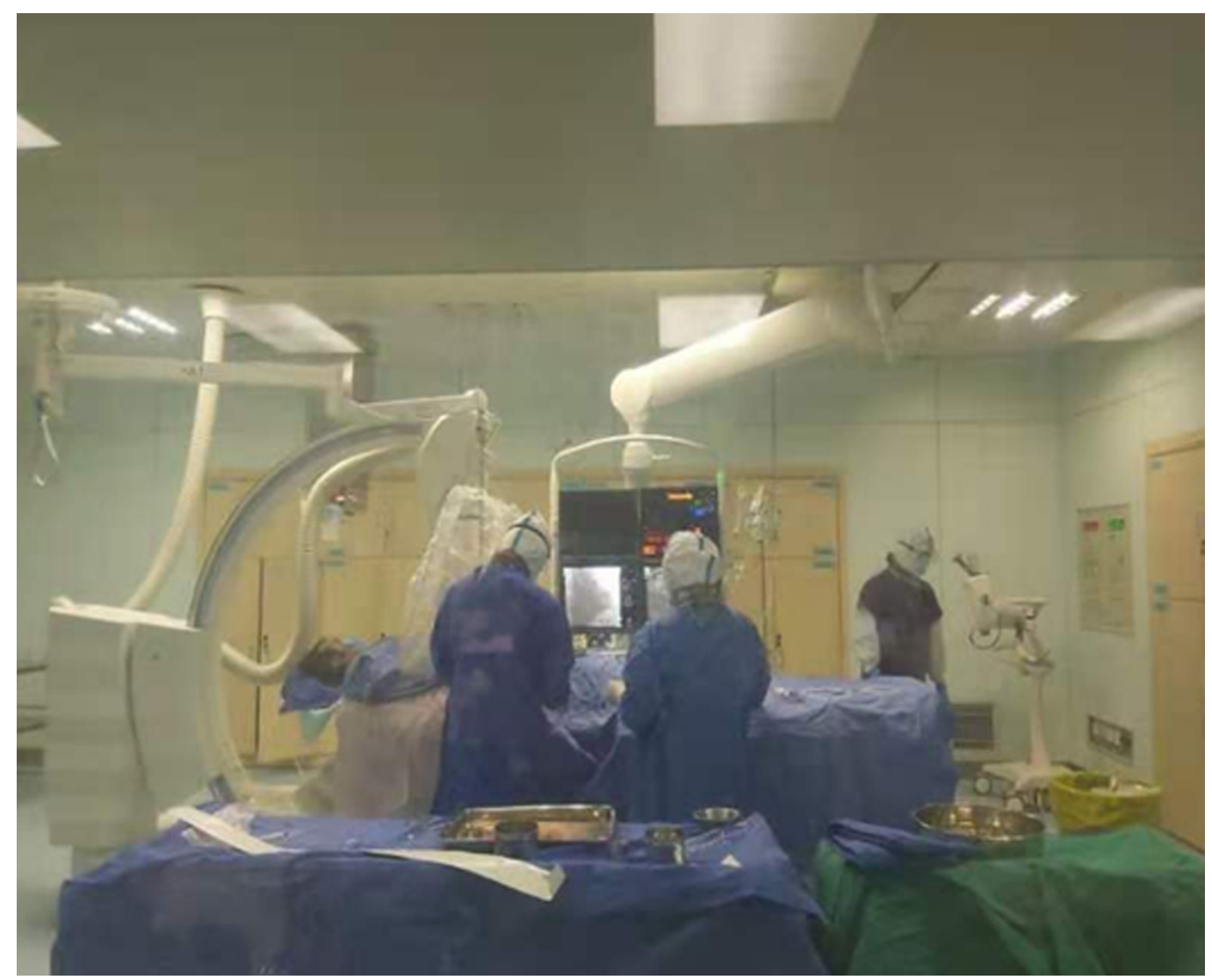

Fig. 1. During the COVID-19 outbreak, primary percutaneous coronary intervention was performed under third-grade protection in the negative-pressure catheter lab of our center for patients with confirmed or uncertain status of SARS-COV-2 infection.

vice on the important time points and clinical outcomes of STEMI patients undergoing PPCI through the contrast with patients visiting outside the COVID-19 outbreak time period.

\section{Methods}

\subsection{Study design and participants}

This single-center retrospective survey included all patients with STEMI admitted to our medical center from January 22, 2020 (the start time of modified CPC operating process implementation) to April 30, 2020 (after lifting the lockdown in Wuhan), and patients who were diagnosed with STEMI and underwent PPCI in our hub during the corresponding time period of previous year (January 22 to April $30,2020)$ were enrolled as a control group. Patients included in this analysis met the following criteria: (1) patients who were clinical diagnosed as acute myocardial infarction with persistent ST-segment arch-like elevation in at least two contiguous electrocardiogram (ECG) leads or new bundle branch blocks (ischaemic repolarization patterns), together with rise of cardiac troponin (cTn) values with at least one above the 99th percentile of the upper reference limit [12]; (2) all those STEMI patients with complete clinical records and followup data who received PPCI in our medical center (including percutaneous coronary balloon dilation, thrombus aspiration and stent implantation). The exclusion criterions were set as follows: (1) patients with incomplete clinical data; (2) non-ST segment elevation acute coronary syndrome (NSTE-ACS); (3) Infarct-related artery (IRA) recanalization or with a functional collateral supply, or triple-vessel disease requiring bypass surgery.

This research was approved by the Medical Ethics Committee of General Hospital of Central Theater Command and followed the principles of Declaration of Helsinki. Informed consent was obtained from all registered patients before discharge to access their anonymous clinical data.

\subsection{Reorganization of CPC operating procedures during COVID-19 outbreak}

To minimize the possible nosocomial cross-infection of SARS-COV-2, we formulated and implemented a detailed protocol for CPC as summarized in Fig. 1. For patients visiting during the outbreak, screening for COVID-19 was conducted upon arrival at the CPC including epidemiological history tracing, body temperature monitoring, and chest imaging examination. In addition, SARS-COV-2 nucleic acid testing was required as soon as possible except for patients in need of emergent reperfusion therapy, and all patients with emergent diseases were managed as suspected COVID19 cases in our hub (isolated in a single-bed unit) until the reporting of nucleic acid detection results. All confirmed 
patients with COVID-19 were transferred to the dedicated quarantine ward with negative-pressure ventilation. During COVID-19 outbreak, the overall principles of our CPC were epidemic prevention and control as top priorities, and all efforts should be taken to ensure timely reperfusion for STEMI patients. PPCI remained the preferred reperfusion strategy if feasible for STEMI patients with uncertain or negative COVID-19 status (without suspected symptoms and close contact history). If the patient with STEMI was confirmed or suspected SARS-COV-2 infectious pneumonia and fibrinolysis was not contraindicated (within the time window of 12 hours), thrombolytic therapy then became the first-line strategy, except for those with high risks of bleeding or disseminated intravascular coagulation (DIC). For SARS-COV2 positive high-risk patients with hemodynamic instability or fatal arrhythmias associated with STEMI, PPCI remained the first-line choice.

All health-care workers who engaged in the interventional (or other aerosol-generating) procedures should be totally protected under the third-grade PPE (including disposable cap, work clothes, double gloves, single-use protective clothing, N95 or better particulate protective masks or powered air filter respirator, protective facial screens or goggles, protective boots, water-proof disposable gown and shoe covers, quick-drying hand disinfectant) [13]. Medical staff who generally contacted with confirmed or suspected patients such as transferring or conservative treatment were protected under grade 2 PPE (including disposable cap and gloves, work clothes, single-use protective clothing, N95 respirator, protective facial screens or goggles, disposable shoe covers, quick-drying hand disinfectant). Grade 1 protection (including disposable cap and gloves, surgical mask or N95 respirator, work clothes) applied to staff in clean or transition zone of emergency department. All associated staff completed pre-training on the correct and appropriate use of PPE to mitigate the risk of nosocomial cross-infection (as shown in Fig. 1).

Patients admitted to our CPC outside the COVID-19 pandemic period were treated in accordance with the routine protocol of Chinese Society of Cardiology in which PPCI was recommended as the first-line treatment strategy for STEMI patients [14].

\subsection{Protection measures of CCL and in-hospital transportation}

For patients with confirmed or uncertain status of COVID-19 infection receiving emergent PPCI, preestablished protocols to prevent cross-contamination between health-care staff and patients were necessary, which included preoperative preparation, peri-operative management, and postoperative terminal disinfection.

1) Preoperative preparation: (1) Notify the staff of CCL receiving patients with confirmed/suspected COVID-19 infection. (2) Open the dedicated pathway for the movement of possible COVID-19 positive patients and close the remaining channels on both sides of the CCL. (3) Turn on the negativepressure ventilation device of CCL. (4) Provide double-layer disposable sterile cover for all the equipment used during the interventional procedure such as $\mathrm{C}$-arm angiographic system and operative bed, etc. (5) Remind the patients without dyspnea to wear a surgical mask during the seamless transport, and only the staff involving in the operation remained in the CCL and equipped with third-grade PPE.

2) Peri-operative management: (1) Keep all the gates of CCL closed and post COVID-19 signage to prevent the entry of unrelated staff. (2) Ensure adequate supplies and equipment in the dedicated counter for COVID-19 and avoid staff entering or leaving the CCL during the operation (before changing PPE). (3) The operation should be as simple and gentle as possible to prevent blood or other body fluids from splashing and causing cross-contamination, whilst maintaining the best clinical outcome for STEMI patients.

3) Postoperative management and terminal disinfection: (1) The cathlab staff doffed the outermost layer protective clothing and retained the grade 2 PPE to transfer patients to a dedicated isolation care unit for further treatment and SARSCOV-2 nucleic acid testing. If the patients were tested positive after the operation, they would be transferred to quarantine ICU designated for COVID-19 patients, and patients would be treated in the coronary care unit (CCU) or singlebed ward if tested negative. (2) Steps for operating staff removing PPE: first take off gloves in the operating room, and then quickly wash hands and wear new gloves. Next, take off goggles or facial screens. After hand disinfection again, take off the protective clothing and foot covers, and placed them in the yellow medical trash collection bags. After entering the buffer (transition) region, doff radio-protective lead clothe and caps into the dedicated container. Replace surgical masks and then enter the clean region where staff could take a shower and change work clothes before leaving the CCL. (3) vTerminal disinfection of CCL and dedicated pathway: the operating room, control room and transfer pathway would be sprayed with hydrogen peroxide (3\%) for air disinfection after the interventional operation. The contaminating material on the floor, wall, instruments or other surfaces should be completely removed and wiped with $2000 \mathrm{mg} / \mathrm{L}$ effective chlorinated disinfectant solution for 30 minutes before wipe off with clean water. Finally, CCL and dedicated pathways would be sterilized by ultraviolet radiation.

\subsection{Outcome measures and data collection}

All clinical data were collected by screening the electronic medical system of our hub. The primary objective of this respective study was to evaluate the change in key time points of reperfusion therapy for STEMI patients during the COVID19 period compared with the control period, including the median time from symptom onset to first medical contact (SO-to-FMC), the median time from first medical contact to entering cardiac catheterization laboratory (FMC-to-CCL), the door to balloon time (D2B), etc. Secondary objectives of the present study included length of hospital stay, PPCIrelated data, clinical adverse events and outcomes. In addition, all patients were followed up within a six-month 
Table 1. Baseline characteristics of STEMI patients

\begin{tabular}{|c|c|c|c|c|c|}
\hline \multirow{2}{*}{ Parameters } & Total & COVID-era group & Control group & Statistics & \multirow{2}{*}{$P$ values } \\
\hline & $(n=62)$ & $(n=21)$ & $(n=41)$ & $\left(\chi^{2} / Z\right)$ & \\
\hline Male gender, $n(\%)$ & $50(80.6)$ & $19(90.5)$ & $31(75.6)$ & 1.129 & 0.288 \\
\hline Age (year), $M(Q L, Q U)$ & $59.0(48.5,69.3)$ & $55.0(45.5,70.0)$ & $59.0(49.0,68.5)$ & -0.618 & 0.537 \\
\hline Site of MI, $n(\%)$ & & & & - & 0.484 \\
\hline Anterior wall & $25(40.3)$ & $7(33.3)$ & $18(43.9)$ & & \\
\hline Inferior wall & $15(24.2)$ & $4(19.0)$ & $11(26.8)$ & & \\
\hline Inferior + RV/Posterior wall & $15(24.2)$ & $6(28.6)$ & $9(22.0)$ & & \\
\hline Others & $7(11.3)$ & $4(19.0)$ & $3(7.1)$ & & \\
\hline Hypertension, $n$ (\%) & $35(56.5)$ & $13(61.9)$ & $22(53.7)$ & 0.384 & 0.535 \\
\hline Hyperglycemia, $n$ (\%) & $36(58.1)$ & $13(61.9)$ & $23(56.1)$ & 0.192 & 0.661 \\
\hline Hyperlipidemia, $n$ (\%) & $31(50.0)$ & $9(42.9)$ & $22(53.7)$ & 0.648 & 0.421 \\
\hline Hyperuricemia, $n$ (\%) & $16(25.8)$ & $6(28.6)$ & $10(24.4)$ & 0.127 & 0.722 \\
\hline Smoking, $n(\%)$ & $31(50.0)$ & $8(38.1)$ & $23(56.1)$ & 1.800 & 0.180 \\
\hline Family history of CVD, $n(\%)$ & $11(17.7)$ & $3(14.3)$ & $8(19.5)$ & 0.025 & 0.874 \\
\hline Route of hospitalization, $n(\%)$ & & & & - & 0.556 \\
\hline Self-admission & $56(90.3)$ & $19(90.5)$ & $37(90.2)$ & & \\
\hline Transfer admission & $5(8.1)$ & $1(4.8)$ & $4(9.8)$ & & \\
\hline Onset in hospital & $1(1.6)$ & $1(4.8)$ & 0 & & \\
\hline
\end{tabular}

period through the outpatient system, WeChat, telephone, etc. Major adverse cardiovascular events (MACE) within 6 months after discharge was defined as malignant arrhythmia, re-admission due to heart failure, recurrent myocardial infarction or angina, and all-cause death.

\subsection{Statistical analysis}

Continuous variables were expressed as median and interquartile ranges ( $1^{\text {st }}$ and $3^{\text {rd }}$ quartiles), and compared by Mann-Whitney U test. Categorical variables were expressed as frequencies (percentages), and compared using the Chisquare test or Fisher's exact test as appropriate. A two-tailed $P$ value less than 0.05 was considered as statistically significant. All statistical analyses were performed using SPSS (version 26.0 for Windows; SPSS Inc., Chicago, Illinois, USA).

\section{Results \\ 3.1 Baseline characteristics}

A total of 62 patients with the diagnosis of STEMI receiving PPCI in our hub were included in this study. Among them, 21 patients visited during the COVID-19 outbreak period and none of them tested positive for SARS-COV-2. In the same period of 2019,41 patients presented to our hub underwent PPCI for STEMI in our hospital. As expected, the number of patients receiving PPCI significantly reduced during the study period as compared to control period. Age, gender, sites of infracted lesions on electrocardiogram (ECG), cardiovascular risk factors, and route of hospital admission were similar between the COVID-19 era group and the control group $(P>0.05)$. Baseline demographic and clinical characteristics of included patients are summarized in Table 1 .

\subsection{Key time points of reperfusion therapy for STEMI patients in CPC}

As shown in Table 2, the median time from symptom onset to first medical contact (SO-to-FMC) in the COVID-19 era group was nominally longer as compared to the control group ( $4.35 \mathrm{~h}$ vs. $2.58 \mathrm{~h}, P=0.258$ ), and the proportion of patients with SO-to-FMC time $>6 \mathrm{~h}$ was significantly higher in the COVID-19 era group than the ratio observed in 2019 (47.6\% vs. $22.0 \%, P=0.038$ ). The median time from first medical contact to entering cardiac catheterization laboratory (FMC-to-CCL), time from entering cardiac catheterization laboratory to puncture (CCL-to-P) and the door to balloon time (D2B) in the COVID-19 era group were significantly longer compared with the control group (65.0 min vs. 49.0 $\min , P=0.020$; $10.0 \mathrm{~min}$ vs. $8.0 \mathrm{~min}, P=0.016 ; 87.0 \mathrm{~min}$ vs. $70.0 \mathrm{~min}, P=0.016$, respectively), which were possibly related to the reorganization of CPC operating procedures. No significant differences in other key time nodes were observed between the two groups.

\subsection{Procedure characteristics}

The characteristics of PPCI were shown in Table 3. There were no significant differences in puncture approach, infarctrelated artery (IRA), Thrombolysis in Myocardial Infarction (TIMI) flow grade before and after the procedure, and stent type between the two groups $(P>0.05)$. A final TIMI flow grade III was achieved in all patients $(62 / 62,100 \%)$ of the two groups after the interventional procedures, which indicated that reorganization of CPC had no significant impact on the opportunity for STEMI patients to receive effective reperfusion therapy. 
Table 2. Comparison of important time points for reperfusion therapy between two groups

\begin{tabular}{lccccc}
\hline \multirow{2}{*}{ Parameters } & Total & COVID-era group & Control group & Statistics & $P$ values \\
\cline { 2 - 5 } & $(n=62)$ & $(n=21)$ & $(n=41)$ & $\left(\chi^{2} / Z\right)$ & \\
\hline SO-to-FMC $(\mathrm{h}), M\left(Q_{L}, Q_{U}\right)$ & $2.63(1.42,6.42)$ & $4.35(1.34,9.00)$ & $2.58(1.31,5.81)$ & -1.130 & 0.258 \\
SO-to-FMC $>6 \mathrm{~h} n(\%)$ & $19(30.6)$ & $10(47.6)$ & $9(22.0)$ & 4.305 & 0.038 \\
SO-to-FMC $>12 \mathrm{~h} n(\%)$ & $6(9.7)$ & $3(14.3)$ & $3(7.3)$ & 0.180 & 0.671 \\
FMC-to-ECG $(\min ), M\left(Q_{L}, Q_{U}\right)$ & $4.0(3.0,5.0)$ & $4.0(3.5,5.0)$ & $4.0(3.0,5.0)$ & -1.766 & 0.077 \\
FMC-to-Drugs $(\min ), M\left(Q_{L}, Q_{U}\right)$ & $8.0(7.0,10.0)$ & $8.0(7.0,10.0)$ & $8.0(6.0,9.5)$ & -0.781 & 0.435 \\
FMC-to-CCL (min), $M\left(Q_{L}, Q_{U}\right)$ & $52.5(44.8,66.5)$ & $65.0(50.0,83.0)$ & $49.0(43.5,57.5)$ & -2.330 & 0.020 \\
CCL-to-P $(\min ), M\left(Q_{L}, Q_{U}\right)$ & $8.0(6.0,10.0)$ & $10.0(7.5,11.0)$ & $8.0(6.0,9.5)$ & -2.399 & 0.016 \\
D2B (min), $M\left(Q_{L}, Q_{U}\right)$ & $75.0(66.8,88.3)$ & $87.0(76.0,108.0)$ & $70.0(63.5,81.5)$ & -2.969 & 0.003 \\
D2B $>90 \min n(\%)$ & $14(22.6)$ & $7(33.3)$ & $7(17.1)$ & 1.273 & 0.259 \\
\hline
\end{tabular}

CCL-to-P, Time from entering cardiac catheterization laboratory to puncture; D2B, Time from entering the door to ballon; FMC-to-CCL, Time from first medical contact to entering cardiac catheterization laboratory; FMC-to-Drugs, Time From first medical contact to the package of medicine for MI; FMC-to-ECG, Time from first medical contact to first ECG; $M\left(Q_{L}, Q_{U}\right)$, Median (lower quartile, upper quartile); SO-to-FMC, Time from symptom onset to first medical contact.

Table 3. Procedure characteristics and outcomes

\begin{tabular}{|c|c|c|c|c|c|}
\hline \multirow{2}{*}{ Parameters } & Total & COVID-era group & Control group & Statistics & \multirow{2}{*}{$P$ values } \\
\hline & $(n=62)$ & $(n=21)$ & $(n=41)$ & $\left(\chi^{2} / Z\right)$ & \\
\hline PPCI approach, $n(\%)$ & & & & 2.201 & 0.138 \\
\hline via radial artery & $46(74.2)$ & $18(85.7)$ & $28(68.3)$ & & \\
\hline via femoral artery & $16(25.8)$ & $3(14.3)$ & $13(31.7)$ & & \\
\hline IRA, $n(\%)$ & & & & - & 0.525 \\
\hline LAD & $26(41.9)$ & $8(38.1)$ & $18(43.9)$ & & \\
\hline LCX & $5(8.1)$ & $1(4.8)$ & $4(9.8)$ & & \\
\hline RCA & $30(48.4)$ & $11(52.4)$ & $19(46.3)$ & & \\
\hline $\mathrm{LAD}+\mathrm{LCX}$ & $1(1.6)$ & $1(4.8)$ & 0 & & \\
\hline TIMI flow grade before Ballon, $n(\%)$ & & & & 2.070 & 0.150 \\
\hline 0 class & $48(77.4)$ & $19(90.5)$ & $29(70.7)$ & & \\
\hline I IIclass & $14(22.6)$ & $2(9.5)$ & $12(29.3)$ & & \\
\hline \multicolumn{6}{|l|}{ TIMI flow grade after PPCI, $n(\%)$} \\
\hline III class & $62(100)$ & $21(100)$ & $41(100)$ & - & - \\
\hline DES, $n(\%)$ & & & & - & 0.914 \\
\hline 0 & $6(9.7)$ & $2(9.5)$ & $4(9.8)$ & & \\
\hline Single & $41(66.1)$ & $13(61.9)$ & $28(68.3)$ & & \\
\hline$\geq 2$ & $15(24.2)$ & $6(28.6)$ & $9(22.0)$ & & \\
\hline
\end{tabular}

DES, Drug-eluting stent; IRA, Infarct-related artery; LAD, Left anterior descending artery; LCX, Left circumflex artery; RCA, Right coronary artery; TIMI, Thrombolysis in myocardial infarction.

\subsection{In-hospital adverse events and length of stay}

The in-hospital adverse events and related indicators were shown in Table 4. There was no significant difference in the proportion of heart failure (Killip classification $\geq$ II) and arrhythmia during hospitalization between the two groups $(P>0.05)$. However, the ratio of cardiogenic shock was significantly higher in the COVID-19 era group (47.6\% vs. $19.5 \%, P=0.021$ ), which may be due to delayed reperfusion. The ratio of thromboembolic events was numerically higher in the COVID-19 era group compared with control group, which did not reach statistical significance (14.3\% vs. $2.4 \%$, $P=0.108$ ). Transthoracic echocardiographic findings during hospitalization were similar between two groups (partial parameters were not measured in some patients as shown in Table 4), and the median levels of serum $\mathrm{N}$-terminal prohor- mone of B-type natriuretic peptide (NT-proBNP), Creatine kinase-MB (CK-MB), and cardiac troponin $\mathrm{T}(\mathrm{cTnT})$ were numerically higher in the COVID-19 era group, but no statistically significant differences were observed (all $P$ values $>$ 0.05). No patient died during the initial hospitalization and the length of hospital stay was similar between both groups (median 12 days, $P=0.863$ ).

\subsection{The incidence of MACE during the 6-month post-operative follow up}

There was no patient loss to follow-up during 6-month postoperative period. Patients admitted in 2020 had a numerically higher proportion of overall MACE after discharge as compared to control group ( $14.3 \%$ vs. $2.4 \%)$, but no statistically significant differences were observed $(P=0.211)$. 
Table 4. In-hospital adverse events and related indicators of STEMI patients

\begin{tabular}{|c|c|c|c|c|c|}
\hline \multirow{2}{*}{ Parameters } & Total & COVID-era group & Control group & Statistics & \multirow{2}{*}{$P$ values } \\
\hline & $(n=62)$ & $(n=21)$ & $(n=41)$ & $\left(\chi^{2} / Z\right)$ & \\
\hline Heart failure $n(\%)$ & $29(46.8)$ & $13(61.9)$ & $16(39.0)$ & 2.920 & 0.087 \\
\hline Cardiogenic shock $n$ (\%) & $18(29.0)$ & $10(47.6)$ & $8(19.5)$ & 5.325 & 0.021 \\
\hline Arrhythmia $n(\%)$ & $16(25.8)$ & $6(28.6)$ & $10(24.4)$ & 0.127 & 0.722 \\
\hline Sinus bradycardia & $7(11.3)$ & $2(9.5)$ & $5(12.2)$ & & \\
\hline AVB & $2(3.2)$ & $1(4.8)$ & $1(2.4)$ & & \\
\hline BBB & $3(4.8)$ & $2(9.5)$ & $1(2.4)$ & & \\
\hline VF & $2(3.2)$ & $1(4.8)$ & $1(2.4)$ & & \\
\hline $\mathrm{AF}$ & $2(3.2)$ & & $2(9.5)$ & & \\
\hline Thromboembolic events, $n$ (\%) & $4(6.5)$ & $3(14.3)$ & $1(2.4)$ & 3.229 & 0.108 \\
\hline NT-proBNP (pg/mL), $M\left(Q_{L}, Q_{U}\right)$ & $1238.0(521.0,2710.8)$ & $1846.0(970.0,3295.5)$ & $1070.0(420.0,2466.0)$ & -1.391 & 0.164 \\
\hline CK-MB $(\mathrm{ng} / \mathrm{mL}), M\left(Q_{L}, Q_{U}\right)$ & $171.7(93.8,237.0)$ & $209.1(109.9,267.9)$ & $163.1(89.8,227.2)$ & -1.259 & 0.208 \\
\hline $\mathrm{CTnT}(\mathrm{ng} / \mathrm{mL}), M\left(Q_{L}, Q_{U}\right)$ & $4.78(2.78,8.14)$ & $5.23(2.98,9.22)$ & $4.42(2.71,7.96)$ & -0.761 & 0.447 \\
\hline \multicolumn{6}{|l|}{ TTE } \\
\hline $\operatorname{LVEF}(\%), M\left(Q_{L}, Q_{U}\right)$ & $63.0(55.0,66.0)$ & $63.0(55.0,63.5)$ & $63.0(57.0,66.0)$ & 0.853 & 0.393 \\
\hline $\mathrm{FS}(\%), M\left(Q_{L}, Q_{U}\right)$ & $34.8(33.0,37.0)$ & $34.0(28.6,37.0)$ & $35.4(33.6,37.1)$ & -1.224 & 0.221 \\
\hline $\operatorname{LVESV}(\mathrm{mL}), M\left(Q_{L}, Q_{U}\right)$ & $41.0(32.2,49.6)$ & $41.0(32.3,58.7)$ & $41.2(32.2,45.9)$ & -0.471 & 0.638 \\
\hline $\operatorname{LVEDV}(\mathrm{mL}), M\left(Q_{L}, Q_{U}\right)$ & $107.0(90.0,119.0)$ & $106.0(94.5,123.0)$ & $108.0(87.8,118.0)$ & -0.046 & 0.964 \\
\hline RWMA, $n(\%)$ & $36(59.0,36 / 61)$ & $15(71.4)$ & $21(52.5,21 / 40)$ & 2.040 & 0.153 \\
\hline Aneurysm, $n(\%)$ & $8(13.1,8 / 61)$ & $3(14.3)$ & $5(12.5,5 / 40)$ & 0.000 & 1.000 \\
\hline Hospital stay (d), $M\left(Q_{L}, Q_{U}\right)$ & $12.0(10.0,14.0)$ & $12.0(9.5,14.5)$ & $12.0(10.5,14.0)$ & -0.173 & 0.863 \\
\hline
\end{tabular}

AF, Atrial fibrillation; AVB, Atrioventricular block; BBB, Bundle branch block; CK-MB, Creatine kinase-MB; CTnT, Cardiac troponin T; FS, Fraction Shortening; LEEDV, Left ventricular end-diastolic volume; LVEF, Left ventricular ejection fraction; LVESV, Left ventricular end systolic volume; $M\left(Q_{L}, Q_{U}\right)$, Median (lower quartile, upper quartile); NT-proBNP, N-terminal pro B-type natriuretic peptide; RWMA, Regional wall motion abnormality; TTE, Transthoracic echocardiography; VF, Ventricular fibrillation.

Table 5. MACE data between two groups during the 6-month post-operative follow up

\begin{tabular}{lccccc}
\hline \multirow{2}{*}{ Parameters } & Total & COVID-era group & Control group & Statistics & \multirow{2}{*}{$P$ values } \\
\cline { 2 - 5 } & $(n=62)$ & $(n=21)$ & $(n=41)$ & $\left(\chi^{2} / Z\right)$ & \\
\hline MACE $n$ (\%) & $4(6.5)$ & $3(14.3)$ & $1(2.4)$ & 1.565 & 0.211 \\
Heart failure & $1(1.6)$ & $1(4.8)$ & 0 & & \\
Recurrent angina & $2(3.2)$ & $1(4.8)$ & $1(2.4)$ & & \\
Malignant arrhythmia & $1(1.6)$ & $1(4.8)$ & 0 & & \\
\hline
\end{tabular}

During the COVID-19 era period, 3 patients $(14.3 \%, 3 / 21)$ were re-admitted with persistent ventricular tachycardia, recurrent heart failure and unstable angina respectively. In the control group, 1 patient $(2.4 \%, 1 / 41)$ was re-admitted for recurrent unstable angina by 3 months after discharge. All readmitted patients recovered after further conservative treatment and there were no disease-related mortalities during the follow-up period. MACE data after discharge were shown in Table 5.

\subsection{Nosocomial cross-infection of SARS-COV-2 in CPC}

All relevant medical staff and STEMI patients were tested negative for the nucleic acid of SARS-COV-2 via reverse transcription-polymerase chain reaction (RT-PCR) during the study period. There was no in-hospital cross-infection of COVID-19 after the reorganization of our hub.

\section{Discussion}

This single-center retrospective study indicated that since the COVID-19 outbreak in Wuhan, the number of STEMI patients who were treated in our CPC and received PPCI was significantly decreased as compared to the analogous time period in 2019. Delays in the presentation of patients from symptom onset to first medical contact were observed in the COVID-19 era group, which might lead to delays in reperfusion therapy. In addition, our CPC modified the conventional operating procedures for STEMI patients admitted during the pandemic to reduce the risk of nosocomial cross-contamination, which would also potentially influence the timing and effectiveness of reperfusion and the prognosis of STEMI patients. In our study, the D2B time in the COVID-19 era group were significantly extended by $17 \mathrm{~min}$ compared with control group, and the SO-to-FMC time extended by $1.77 \mathrm{~h}$, which was longer than the data $(0.89 \mathrm{~h})$ from China Chest Pain Center (CCPC) reported by Xiang 
et al. [10]. These may result from the severity of outbreak and the level of lockdown in Wuhan during the early stage of the pandemic. The proportion of patients with SO-to-FMC time $>6$ hours increased by $25.6 \%$ as compared to the 2019 group in our study, hinting that patients might be unwilling to visit the emergency department due to social distancing or fear of cross-contamination in the hospital and appropriate strategies should be taken to timely identified high-risk groups, such as Telemedicine, etc.

Similar results were publicly reported by several CCL around the world. Garcia et al. analyzed the tendency of PPCI for STEMI patients in 9 high-volume CCL centers of U.S. during the COVID-19 period, which showed a decrease in the monthly rate of patients with STEMI receiving PPCI of 38\% (95\% CI: 26\% 49\%) after March 1, 2020 (the time when the first COVID-19 case reported in New York) [15]. The data from a tertiary cardiac center in U.K. reported by Wilson et al. [16] indicated a decline of roughly 50\% in both CCL activations and STEMI admissions after March 18, 2020 (restriction policy activated). A single-center, prospective study from a regional cardiac center in Milan, Italy [11] showed that $80.8 \%$ STEMI patients underwent PPCI and 19.2\% received conservative treatment due to late presentation during COVID-19 pandemic compared with $100 \%$ patients receiving PPCI in the same period of previous years, and doorto-reperfusion time was 20 min longer in COVID-19 era. Potential reasons for the reduction of PPCI activations for STEMI patients include strict social distancing policy, misdiagnosis in lower-level hospitals, increased use of thrombolytic therapy and medical resources diverted for COVID19 , etc. [17]. In addition, patients would be reluctant to seek medical care in the COVID-19 epidemic area due to fear of contracting SARS-COV-2, which could potentially lead to this decline and impact adversely on the prognosis of patients with STEMI.

The modified reperfusion strategies of CCPC, which recommended thrombolytic therapy as the first-line reperfusion therapy for STEMI patients with uncertain COVID-19 status in the COVID-19 epidemic area if feasible (within the time window of 12 hours and without contraindications), could also influence the timeliness and efficiency of reperfusion treatment [18]. PPCI delay was in the COVID-19 era due to relevant screening and protective measures, etc. Timely PPCI (D2B within $90 \mathrm{~min}$ ) relied on close cooperation among multiple departments. For CPC without independent negative-pressure ventilation CCL or adequate PPE and experiencing interventional staff shortages, initial fibrinolysis therapy may be an optimal choice to prevent in-hospital cross-contamination [19]. Therefore, the risks and benefits of each reperfusion strategy should be re-evaluated depending on the medical conditions of the areas with COVID-19 outbreak [20].

After COVID-19 outbreak in Wuhan, our CPC modified the operating procedures for STEMI patients admitted during the pandemic (Fig. 2). All health-care workers partici- pating in the operation for patients with confirmed or uncertain COVID-19 status were protected under the third-grade PPE and worked in the negative-pressure CCL. The principal aim of our modification was to prevent and control nosocomial cross-infection of SARS-COV-2, and all related departments made every effort to ensure timely reperfusion and salvage the ischaemic myocardium maximally for STEMI patients. During the pandemic, we persisted in PPCI as the preferred strategy for STEMI and attempted to keep the D2B time within $90 \mathrm{~min}$ whenever possible. Although our results showed the median FMC-to-CCL time and D2B time were significantly longer in the COVID-19 era group (delay for approximately 17 minutes), the proportion of STEMI patients who received effective reperfusion therapy for IRA within 90 min remained stable and a final TIMI flow grade III was achieved in all patients.

In our study, the incidence of cardiogenic shock and heart failure during hospitalization increased in the COVID-19 era group, which may be related to the delay in patients' presentation and reperfusion therapy, while there was no significant difference in echocardiographic indicators reflecting ventricular structural remodeling and cardiac function during hospitalization between the two groups. Possible explanations for our results included improvement in ejection fraction after effective reperfusion and the relatively small sample size. The nation-wide data reported by Xiang et al. [10] similarly showed that in-hospital heart failure rate of STEMI patients increased after implementing modified reperfusion strategies in China without available data of left ventricular ejection fraction (LVEF). Wilson et al. [16] reported the significantly lower LVEF of STEMI patients in the COVID-19 era as compared to historical era (43.7\% vs. $47.4 \%)$ and a numerical increase of in-hospital mortality of STEMI during the pandemic, which indicated the potential implications of COVID19 outbreak [21]. The proportion of in-hospital arrhythmia related to myocardial ischemia was similar between the two groups and we found no cases of cardiac arrhythmia associated with COVID-19 [22]. In addition, the rate of thromboembolic events in patients receiving PPCI was numerically higher in the COVID-19 era group (14.3\% vs. $2.4 \%$ ), including deep vein thrombosis and cerebral thromboembolism, etc. Previous literature has reported that hypercoagulability [23] and increased coagulopathy in patients with COVID19 infection [24], which might correlate with SARS-COV-2 mediated microvascular injury [25] and increased the risk of acute coronary syndromes [26]. However, no patients were tested positive for the nucleic acid of SARS-COV-2 during hospitalization in our study and further research would be needed to explore the incidence of coagulopathy in patients admitted for STEMI during the pandemic.

The incidence of MACE during the 6-month postoperative follow up had a slight increase in the COVID-19 era group (14.3\% vs. $2.4 \%$ ) without statistical significance, which required long-term follow up to analyze the unforeseen impact of COVID-19 on the prognosis of STEMI patients [27]. 


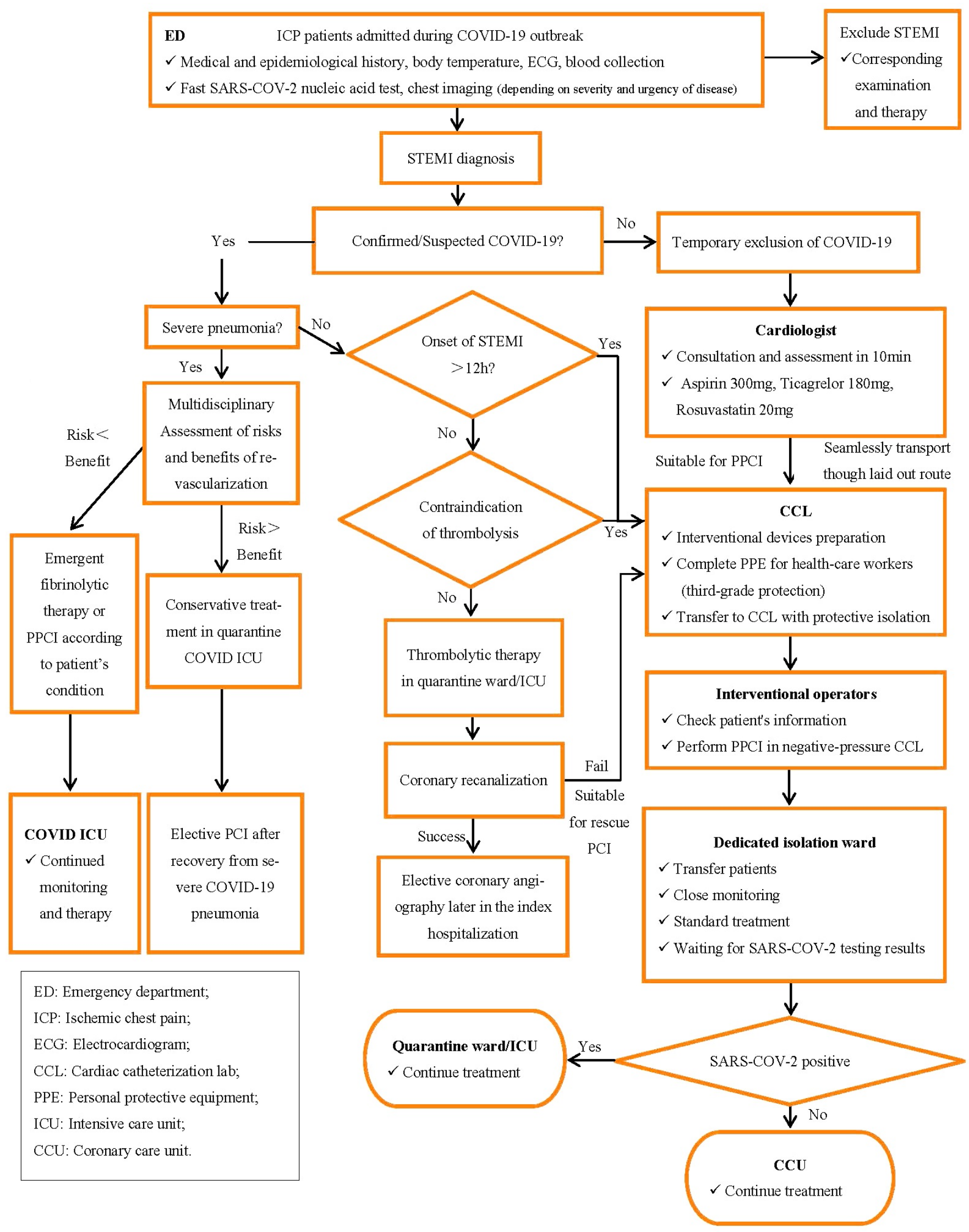

Fig. 2. Flow diagram for the management of patients presenting with STEMI in the context of COVID-19 pandemic. The aim of our strategy was to prevent nosocomial cross-infection of SARS-COV-2, and ensure timely coronary reperfusion. 
This study had several limitations as follows. First, this was a single-center retrospective study with a relatively small sample size, which had limitations inherent to observational studies. Second, the follow-up period was only 6 months, while long-term follow up would affect our results. Last but not least, some unobserved confounders might influence our results. For example, mild patients would be reluctant to seek medical care in the COVID-19 era [28], which tended to worsen the clinical outcomes of the study group. Therefore, our results need to be interpreted cautiously.

\section{Conclusions}

The outbreak of COVID-19 has profoundly affected health-care systems and timely dependent STEMI therapy. In this study, we preliminarily demonstrated the descending trend in STEMI hospitalization and a longer time from symptom onset to first medical contact during COVID-19 pandemic. With a significant delay in D2B time, the incidence of in-hospital cardiogenic shock and heart failure tended to increase in the COVID-19 era. Therefore, rapid adjustment of management strategies for STEMI patients according to prevalence status of epidemic areas could be an effective way to minimize the risks of cross-infection and cardiac collateral damage of COVID-19. Further studies with large sample are needed to explore the potential impact of COVID-19 outbreak on the long-term prognosis of STEMI patients.

\section{Author contributions}

SFD: study design. ZNC, WBF: data collection, analysis. QL, JLW: manuscript drafting. QL, HJL: data analysis and interpretation. SFD: revision of the manuscript.

\section{Ethics approval and consent to participate}

This research was approved by the Medical Ethics Committee of General Hospital of Central Theater Command and followed the principles of Declaration of Helsinki. The clinical registration number of this study is ChiCTR2100042315. Written informed consent was obtained from all registered patients before discharge to access their anonymous clinical data.

\section{Acknowledgment}

We are grateful to all the health-care staff who contributed to the prevention and control of the COVID-19 outbreak.

\section{Funding}

This work was supported by Research Joint Project of Health and Family Planning Commission of Hubei Province (WJ2018H0068).

\section{Conflict of interest}

The authors of this study have no conflicts of interest to declare.

\section{References}

[1] Zhu N, Zhang D, Wang W, Li X, Yang B, Song J, et al. A novel coronavirus from patients with pneumonia in China, 2019. New
England Journal of Medicine. 2020; 382: 727-733.

[2] Sohrabi C, Alsafi Z, O’Neill N, Khan M, Kerwan A, Al-Jabir A, et al. World Health Organization declares global emergency: a review of the 2019 novel coronavirus (COVID-19). International Journal of Surgery. 2020; 76: 71-76.

[3] World Health Organization. Coronavirus disease 2019 (COVID19): situation report, 64. World Health Organization. 2020. Available at: https://apps.who.int/iris/handle/10665/331608 (Accessed: 24 March 2020).

[4] Guan W-J, Ni Z-Y, Hu Y, Liang W-H, Ou C-Q, He J-X, et al. Clinical characteristics of coronavirus disease 2019 in China. New England Journal of Medicine. 2020; 382: 1708-1720.

[5] Huang C, Wang Y, Li X, Ren L, Zhao J, Hu Y, et al. Clinical features of patients infected with 2019 novel coronavirus in Wuhan, China. The Lancet. 2020; 395: 497-506.

[6] Xu Z, Shi L, Wang Y, Zhang J, Huang L, Zhang C, et al. Pathological findings of COVID-19 associated with acute respiratory distress syndrome. The Lancet Respiratory Medicine. 2020; 8: 420422.

[7] Zhou F, Yu T, Du R, Fan G, Liu Y, Liu Z, et al. Clinical course and risk factors for mortality of adult inpatients with COVID-19 in Wuhan, China: a retrospective cohort study. The Lancet. 2020; 395: 1054-1062.

[8] Choy K, Wong AY, Kaewpreedee P, Sia SF, Chen D, Hui KPY, et al. Remdesivir, lopinavir, emetine, and homoharringtonine inhibit SARS-CoV-2 replication in vitro. Antiviral Research. 2020; 178: 104786.

[9] De Filippo O, D’Ascenzo F, Angelini F, Bocchino PP, Conrotto F, Saglietto A, et al. Reduced rate of hospital admissions for ACS during Covid-19 outbreak in Northern Italy. New England Journal of Medicine. 2020; 383: 88-89.

[10] Xiang D, Xiang X, Zhang W, Yi S, Zhang J, Gu X, et al. Management and outcomes of patients with STEMI during the COVID-19 pandemic in China. Journal of the American College of Cardiology. 2020; 76: 1318-1324.

[11] Gramegna M, Baldetti L, Beneduce A, Pannone L, Falasconi G, Calvo F, et al. ST-segment-elevation myocardial infarction during COVID-19 pandemic: insights from a regional public service healthcare hub. Circulation: Cardiovascular Interventions. 2020; 13: e009413.

[12] Ibanez B, James S, Agewall S, Antunes MJ, Bucciarelli-Ducci C, Bueno $\mathrm{H}$, et al. 2017 ESC Guidelines for the management of acute myocardial infarction in patients presenting with ST-segment elevation: the Task Force for the management of acute myocardial infarction in patients presenting with ST-segment elevation of the European Society of Cardiology (ESC). European Heart Journal. 2018; 39: 119-177.

[13] Zhang L, Fan Y, Lu Z. Experiences and lesson strategies for cardiology from the COVID-19 outbreak in Wuhan, China, by 'on the scene' cardiologists. European Heart Journal. 2020; 41: 1788-1790.

[14] Zhang Y, Yu B, Han Y, Wang J, Yang L, Wan Z, et al. Protocol of the China ST-segment elevation myocardial infarction (STEMI) Care Project (CSCAP): a 10-year project to improve quality of care by building up a regional STEMI care network. BMJ Open. 2019; 9: $\mathrm{e} 026362$.

[15] Garcia S, Albaghdadi MS, Meraj PM, Schmidt C, Garberich R, Jaffer FA, et al. Reduction in ST-segment elevation cardiac catheterization laboratory activations in the United States during COVID19 pandemic. Journal of the American College of Cardiology. 2020; 75: 2871-2872.

[16] Wilson SJ, Connolly MJ, Elghamry Z, Cosgrove C, Firoozi S, Lim P, et al. Effect of the COVID-19 pandemic on ST-segmentelevation myocardial infarction presentations and in-hospital outcomes. Circulation: Cardiovascular Interventions. 2020; 13: e009438.

[17] Huynh K. Reduced hospital admissions for ACS - more collateral damage from COVID-19. Nature Reviews Cardiology. 2020; 17 : 453-453. 
[18] Zeng J, Huang J, Pan L. How to balance acute myocardial infarction and COVID-19: the protocols from Sichuan Provincial People's Hospital. Intensive Care Medicine. 2020; 46: 1111-1113.

[19] Mahmud E, Dauerman HL, Welt FGP, Messenger JC, Rao SV, Grines C, et al. Management of acute myocardial infarction during the COVID-19 pandemic: a consensus statement from the Society for Cardiovascular Angiography and Interventions (SCAI), the American College of Cardiology (ACC), and the American College of Emergency Physicians (ACEP). Catheterization and Cardiovascular Interventions. 2020; 96: 336-345.

[20] Briedis K, Aldujeli A, Aldujeili M, Briede K, Zaliunas R, Hamadeh $A$, et al. Considerations for management of acute coronary syndromes during the SARS-CoV-2 (COVID-19) pandemic. The American Journal of Cardiology. 2020; 131: 115-119.

[21] Hamadeh A, Aldujeli A, Briedis K, Tecson KM, Sanz-Sánchez J, Al dujeili $M$, et al. Characteristics and outcomes in patients presenting with COVID-19 and ST-segment elevation myocardial infarction. The American Journal of Cardiology. 2020; 131: 1-6.

[22] Elsaid O, McCullough PA, Tecson KM, Williams RS, Yoon A. Ventricular fibrillation storm in coronavirus 2019. The American Journal of Cardiology. 2020; 135: 177-180.

[23] Singhania N, Bansal S, Nimmatoori DP, Ejaz AA, McCullough PA,
Singhania G. Current overview on hypercoagulability in COVID19. American Journal of Cardiovascular Drugs. 2020; 20: 393-403.

[24] Mahajan P, Dass B, Radhakrishnan N, McCullough P. COVID-19associated systemic thromboembolism: a case report and review of the literature. Cardiorenal Medicine. 2020; 10: 462-469.

[25] Zhang J, Tecson KM, McCullough PA. Endothelial dysfunction contributes to COVID-19-associated vascular inflammation and coagulopathy. Reviews in Cardiovascular Medicine. 2020; 21: 315.

[26] Kermani-Alghoraishi M. A review of coronary artery thrombosis: a new challenging finding in COVID-19 patients and ST-elevation myocardial infarction. Current Problems in Cardiology. 2020; 323: 100744.

[27] Ruocco G, McCullough PA, Tecson KM, Mancone M, De Ferrari GM, D'Ascenzo F, et al. Mortality risk assessment using CHA(2)DS(2)-VASc scores in patients hospitalized with coronavirus disease 2019 infection. The American Journal of Cardiology. 2020; 137: 111-117.

[28] Moroni F, Gramegna M, Ajello S, Beneduce A, Baldetti L, Vilca LM, et al. Collateral damage: medical care avoidance behavior among patients with myocardial infarction during the COVID-19 pandemic. JACC: Case Reports. 2020; 2: 1620-1624. 